\title{
Seroepidemiology of Infectious Bursal Disease in Poultry Reared Under Backyard Production System in Wolaita Zone, Southern Ethiopia
}

\author{
Mihret Amajo' \\ Asamnew Tesfaye ${ }^{2}$ \\ Teshale Sori ${ }^{3}$ \\ Haileleul Negussie ${ }^{3}$ \\ 'School of Veterinary Medicine, College \\ of Agriculture, Wolaita Sodo University, \\ Wolaita Sodo, Ethiopia; ${ }^{2}$ National Animal \\ Health Diagnostic and Investigation \\ Center, Sebeta, Ethiopia; ${ }^{3}$ Department of \\ Clinical Studies, College of Veterinary \\ Medicine and Agriculture, Addis Ababa \\ University, Bishoftu, Ethiopia
}

Background: Infectious bursal disease (IBD) has been known to cause high morbidity and mortality in chickens resulting in considerable financial losses to poultry producers. This study was performed with the objectives of estimating the seroprevalence and associated risk factors of IBD in backyard chickens in Wolaita zone, southern Ethiopia.

Methods: A total of 482 serum samples were collected from chickens reared under backyard systems using a multi-stage cross-sectional study design. The serum samples were tested for the presence of anti-IBDV antibodies using an indirect enzyme-linked immunosorbent assay (ELISA). A questionnaire survey was also performed to identify risk factors affecting chicken production in the study area.

Results: From the total of 482 serum samples tested, 236 (48.96\%; 95\% CI: 44.32-53.42) were positive for anti-IBDV antibodies. Higher seroprevalence was recorded in Humbo district $(55.75 \%$; 95\% CI: 46.11-65.09) followed by Sodo Zuria (51.54\%; 95\% CI: 42.62 60.39), Damotgale (46.22\%; 95\% CI: 36.49-56.18), and Kindokoysha district (42.86\%; 95\% CI: 34.32-51.72) although the difference was not statistically significant. Significantly lower prevalence was recorded in indigenous chickens (43.36\%; 95\% CI: 37.53-49.32) compared to exotic chickens $(57.14 \%$; 95\% CI: 49.89-64.17). The odds of occurrence of IBD in the local chicken breed was 0.67 times lower than that of the exotic chicken breed. The odds of occurrence of IBD in chickens from flock size $\geq 5$ chickens was 4.33 times higher than chickens from flock size $<5$ chickens. A statistically significant association $(\mathrm{P}<0.05)$ was observed between treatment history and isolation of sick chickens with mortality in the flock. Conclusion: This study revealed that IBD is one of the major infectious diseases that affect the traditionally managed chickens in the study area with the flock size and breed of chickens are identified as important risk factors for IBD occurrence. Besides, chicken producers did not have enough knowledge about the nature and epidemiology of IBD. Thus, proper management practices together with appropriate vaccination programs are necessary to reduce IBD incidence in the study areas.

Keywords: backyard chicken, IBD, risk factors, seroprevalence, Wolaita Sodo, Ethiopia

\section{Introduction}

Poultry production has a great significance in the development of Ethiopia, where they have an essential role in reducing poverty, providing food security, and use as an immediate cash income for the rural communities. ${ }^{1}$ The Ethiopian government identified the poultry sector as a priority area in its endeavor to achieve food security. This, however, requires an understanding of the existing constraints such as infectious
Correspondence: Haileleul Negussie Department of Clinical Studies, College of Veterinary Medicine and Agriculture, Addis Ababa University, P. O. Box: 34, Bishoftu, Ethiopia

Email haileleul.negussie@aau.edu.et 
diseases, which pose considerable setbacks on poultry production. Among the multiple health problems, infectious bursal disease (IBD), also known as Gumboro, is one of the major health constraints that hamper poultry productivity in Ethiopia. $^{2,3}$

Infectious bursal disease is an acute and highly contagious viral infection in young chickens. IBD is caused by infectious bursal disease virus (IBDV), which belongs to the Avibirnavirus genus of the family Birnaviridae. ${ }^{4,5}$ It causes an acute, highly contagious, immunosuppressive disease in chickens. ${ }^{4}$ IBD occurs in one of the two forms, depending on the age of the chicken when it becomes infected. The subclinical form occurs in chickens less than 3 weeks of age, causing permanent and severe immunosuppression. ${ }^{6}$ Most infections that occur in the field are of the subclinical variety, and this is the more economically important form of the disease. The clinical form of the disease occurs in chickens between 3 and 6 weeks of age. ${ }^{6}$ The majority of the lesions are found in the bursa of Fabricius when birds are necropsied. ${ }^{7,8}$

IBD is an emerging disease of chicken in Ethiopia that the disease has been speculated to be introduced concurrently with the importation of chickens to flourish the private large-scale commercial poultry farms in the country. ${ }^{9}$ The first report of IBD in Ethiopia was in 2005 from commercial poultry farms. ${ }^{9}$ Since then, it became among the most significant diseases that affect the poultry industry throughout the country. ${ }^{9-11}$ In recent years, there has been an unexpected increase in the frequency of IBD outbreaks in commercial and backyard poultry production systems. In Ethiopia, backyard chickens are mostly indigenous chicken breeds managed by individual households and maintained under scavenging regimens in the backyards with inadequate housing, feeding, and health care. In recent years, Ethiopia is experiencing rapid growth in the poultry sector. Improved breeds of chickens are distributed all over chickenproducing areas of Ethiopia from breeding and multiplication centers. Poor management practice and limited vaccination programs in traditionally managed chickens facilitate the spread of IBDV. In backyard chicken, poultry diseases including IBD are the major constraints of poultry production. Previous seroepidemiological studies conducted in Ethiopia indicated that IBD is the major health problem of the poultry industry with the seroprevalence reaching up to $83.1 \%$ and a mortality rate ranging from $49.9 \%$ to $75 \%$ in exotic and crossbred chickens. ${ }^{9,11-13}$
Strict biosecurity measures and regular vaccination are the main tools for IBD control. Vaccination of chickens against IBD using attenuated IBDV strain D78 vaccine produced at the National Veterinary Institute of Ethiopia has been implemented throughout the commercial poultry farms in Ethiopia since it was first reported in $2005 .^{9}$ However, the vaccines are not providing full protection against IBDV, which has been evidenced by the occurrence of IBD outbreaks in vaccinated flocks. Besides, the majority of poultry producers living in the rural part of Ethiopia are not practicing regular vaccination of their chickens against IBDV. Wolaita Soddo is one of the chicken-producing areas of Ethiopia. Although the majority of chickens reared in the area are indigenous ones, the number of exotic breeds of chicken in the area is increasing. Hence, disease epidemiological data are necessary to understand its occurrence and the risk factors involved in the area. Therefore, the objective of this study was to estimate the seroprevalence and risk factors of IBD in backyard chickens in selected districts of Wolaita zone, Southern Ethiopia.

\section{Materials and Methods}

\section{Study Area, Population and Design}

This study was conducted in the Wolaita Zone of the Southern Nations Nationalities and People's (SNNP) Regional State of Ethiopia. A multi-stage cross-sectional approach was carried out from December 2020 to May 2021. Four districts namely Damot Gale (midland), Humbo (lowland), Kindokoysha (lowland), and Sodo Zuria (highland) were selected for this investigation (Figure 1). These districts were selected based on their representative agroecology and higher chicken population. From each district, five Kebeles (the smallest administrative unit equivalent to peasant associations) were selected randomly. Then, individual households were randomly selected from each Kebele. Accordingly, a total of 132 households that rear both indigenous chicken ecotypes and exotic breeds were randomly selected for this study.

The chickens considered in this study were indigenous chickens reared under the backyard production system. In addition, exotic chicken breeds reared by the selected households were also included. By considering the development of the bursa of Fabricius, the age of the chicken was categorized into $<3$ weeks and $\geq 3$ weeks of age. We adopted this method of age classification during data collection. History of vaccination was first obtained for each 


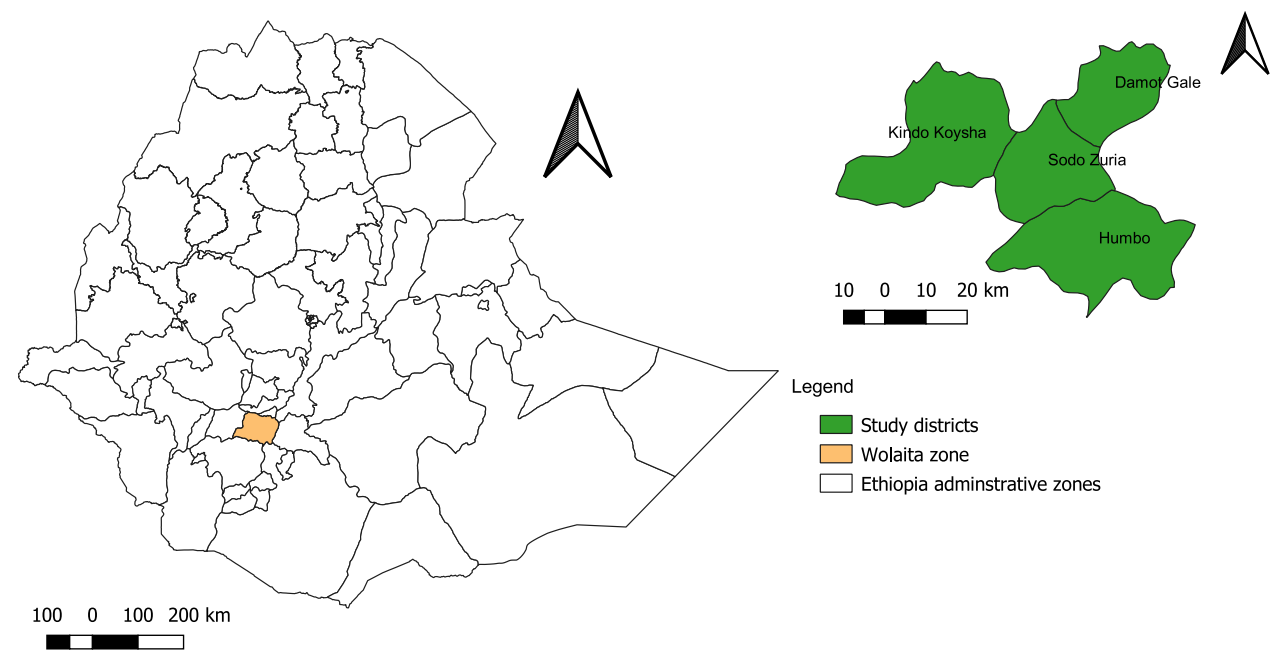

Figure I Map of Ethiopia depicting the location of the study areas. This map was developed from Ethiopian's administrative boundaries shapefile 202I using QGIS version 3.I.I.2.

household and only those unvaccinated chickens against IBD were included. A snapshot survey was done to identify flock size per household and the average was 5 chickens per household. Hence, the flock size was categorized as $\geq 5$ and $<5$ based on the average number of chickens per household.

\section{Sample Size and Sampling Technique}

The minimum sample size required for this study was estimated according to Thrusfield. ${ }^{14}$ An expected prevalence of $82.9 \%$ was considered from the reports of Jenberie et $\mathrm{al}^{11}$ and $95 \%$ confidence interval (CI) and 5\% absolute precision were also considered in the formula. Hence, according to the formula, a minimum of 218 chickens was needed. However, since we used cluster sampling in which all chickens owned by the selected households were included in the sample, a total of 482 chickens made the sample size.

From each chicken, about $2 \mathrm{~mL}$ of blood samples were collected from the wing vein using a disposable syringe and needle. The collected blood samples were kept at room temperature for 2-4 hours to allow the separation of serum from blood clots. The separated sera were transferred to sterile cryovials, labeled individually, and transported to the National Animal Health Diagnostic and Investigation Center (NAHDIC) using an ice-box, where they were stored at $-20^{\circ} \mathrm{C}$ until analyzed.

\section{Serological Analysis}

The serum samples were analyzed for the presence of antiIBDV antibodies using an indirect ELISA kit (IDvet, Louis
Pasteur-Grabels, France) following the manufacturer's instructions. This test kit has diagnostic specificity and sensitivity of $100 \%$ according to the information of the manufacturer's validation data report. BioTek EL800 Microplate Reader was used to measure the optical density (OD) of each sample and control at $450 \mathrm{~nm}$. The results were interpreted based on the sample-to-positive control $\mathrm{OD}$ ratio $(\mathrm{S} / \mathrm{P})$. The results were interpreted as samples with an $\mathrm{S} / \mathrm{P}$ ratio greater than 0.3 and less than or equal to 0.3 were considered positive and negative, respectively.

\section{Questionnaire Survey}

A semi-structured questionnaire was administered to a total of 60 households to obtain relevant information on the features of the backyard poultry management system in the area. The questionnaire was distributed to individuals to fill in relevant data related to poultry production and associated problems. The information gathered with the questionnaire includes the type of poultry production, socioeconomic importance, and management practices.

\section{Data Analysis}

The data obtained in this study were analyzed using $\mathrm{R}$ version 3.6.2. The presence of an association between seropositivity and the potential risk factors considered was analyzed by logistic regression in which the odds ratio was computed to quantify the effects of the risk factors on the occurrence of IBD. A stepwise backward elimination was used to select variables for the final model. The relationship between the predictors and the seroprevalence of IBD was estimated using 
Maximum likelihood. For all analyses, P-values less than 0.05 were considered significant at a $95 \%$ confidence interval.

\section{Results}

\section{Seroprevalence of IBD}

Out of a total of 482 serum samples tested, 236 (48.96\%; 95\% CI: 44.32-53.42) were positive for anti-IBDV antibody. Although the difference was not statistically significant, higher prevalence was observed in Humbo district (55.75\%; 95\% CI: 46.11-65.09) followed by Sodo Zuria (51.54\%; 95\% CI: 42.62-60.39), Damotgale (46.22\%; 95\% CI: 36.49-56.18), and Kindokoysha district (42.86\%; 95\% CI: 34.32-51.72). The prevalence was lower in indigenous chickens $(43.36 \%$; $95 \%$ CI: 37.53-49.32) compared to the exotic ones $(57.14 \%$; 95\% CI: 49.89-64.17) although the difference was not statistically significant (Table 1).

A significantly higher prevalence was observed in chickens tested from the flocks having $\geq 5$ chickens (59.47\%; 95\% CI: 54.02-64.75) compared to flocks having $<5$ chickens $(24.31 \%$; 95\% CI: 17.55-32.15). Chicken belonging to a higher flock size had 4.07 (95\% CI: $2.578-$ 6.43; $\mathrm{P}<0.001)$ times higher Odds of positivity than those from smaller flock sizes as shown in Table 1.

Odds of occurrence of IBD in the local chicken breed were 0.67 times lower than that of the exotic chicken breed. The odd of the occurrence of the IBD in chickens from flock size $\geq 5$ chickens was 4.33 times higher than chicken from flock size $<5$ chickens (Table 1 ).

From the categories of the significant explanatory variable number of flock sizes $\geq 5$ is predicted to have a high probability of IBD occurrence than the categories with $<5$ chickens. As shown in the plot, the predicted probability of IBD in flocks with $\geq 5$ chickens is between 0.55 and 0.65 (Figure 2), which was higher than the predicted probability in flocks with $<5$ chickens (between 0.2 and 0.3 ). The predicted probability of IBD occurrence is also higher in exotic breeds than in local breeds, ranging from 0.3 to 0.65 and 0.25 to 0.55 , respectively (Figure 2).

\section{Questionnaire Survey}

A questionnaire survey was administered to 60 households and the results showed that poultry production is the source of secondary income for the majority of farmers $(81.66 \%)$ and only $8.33 \%$ of respondents used it for home consumption. However, $10 \%$ of the respondents revealed that poultry production is used as the primary source of income. The majority $(73.33 \%)$ of the respondents rear chicken both for egg and meat production; $78.33 \%$ share shelter with chickens whereas $21.66 \%$ provide separate houses for their chicken

Table I Seroprevalence of IBD in Chicken Reared Under Backyard Production System in Wolaita Zone

\begin{tabular}{|c|c|c|c|c|c|c|}
\hline Variable & No. of Tested & No. of Positive & Prevalence (\%) & OR & 95\% Conf. Interval & P-value \\
\hline \multicolumn{7}{|l|}{ District } \\
\hline Damotgale & 106 & 49 & 46.22 & Ref & & \\
\hline Humbo & 113 & 63 & 55.75 & 1.14 & $0.65-2.01$ & 0.644 \\
\hline Kindokoysha & 133 & 57 & 42.85 & 0.89 & $0.5 \mathrm{I}-\mathrm{I} .55$ & 0.679 \\
\hline Sodo Zuria & 130 & 67 & 51.53 & 0.97 & $0.54-1.76$ & 0.922 \\
\hline \multicolumn{7}{|l|}{ Breed } \\
\hline Local & 286 & 124 & 43.35 & 0.69 & $0.45-1.07$ & 0.098 \\
\hline Exotic & 196 & 112 & 57.14 & Ref & & \\
\hline \multicolumn{7}{|l|}{ Sex } \\
\hline Male & 160 & 79 & 49.37 & 0.97 & $0.65-1.46$ & 0.886 \\
\hline Female & 322 & 157 & 48.75 & Ref & & \\
\hline \multicolumn{7}{|l|}{ Age } \\
\hline$<3$ weeks & 102 & 53 & 51.96 & 1.19 & $0.74-1.92$ & 0.474 \\
\hline$\geq 3$ weeks & 380 & 183 & 48.18 & Ref & & \\
\hline \multicolumn{7}{|l|}{ Flock size } \\
\hline$\geq 5$ chicken & 338 & 201 & 59.47 & 4.07 & $2.578-6.43$ & $0.000^{*}$ \\
\hline$<5$ chicken & 144 & 35 & 24.31 & Ref & & \\
\hline Total & 482 & 236 & 48.96 & & $44.52-53.41$ & \\
\hline
\end{tabular}

Note: *Represent statistically significant.

Abbreviation: Ref, reference. 

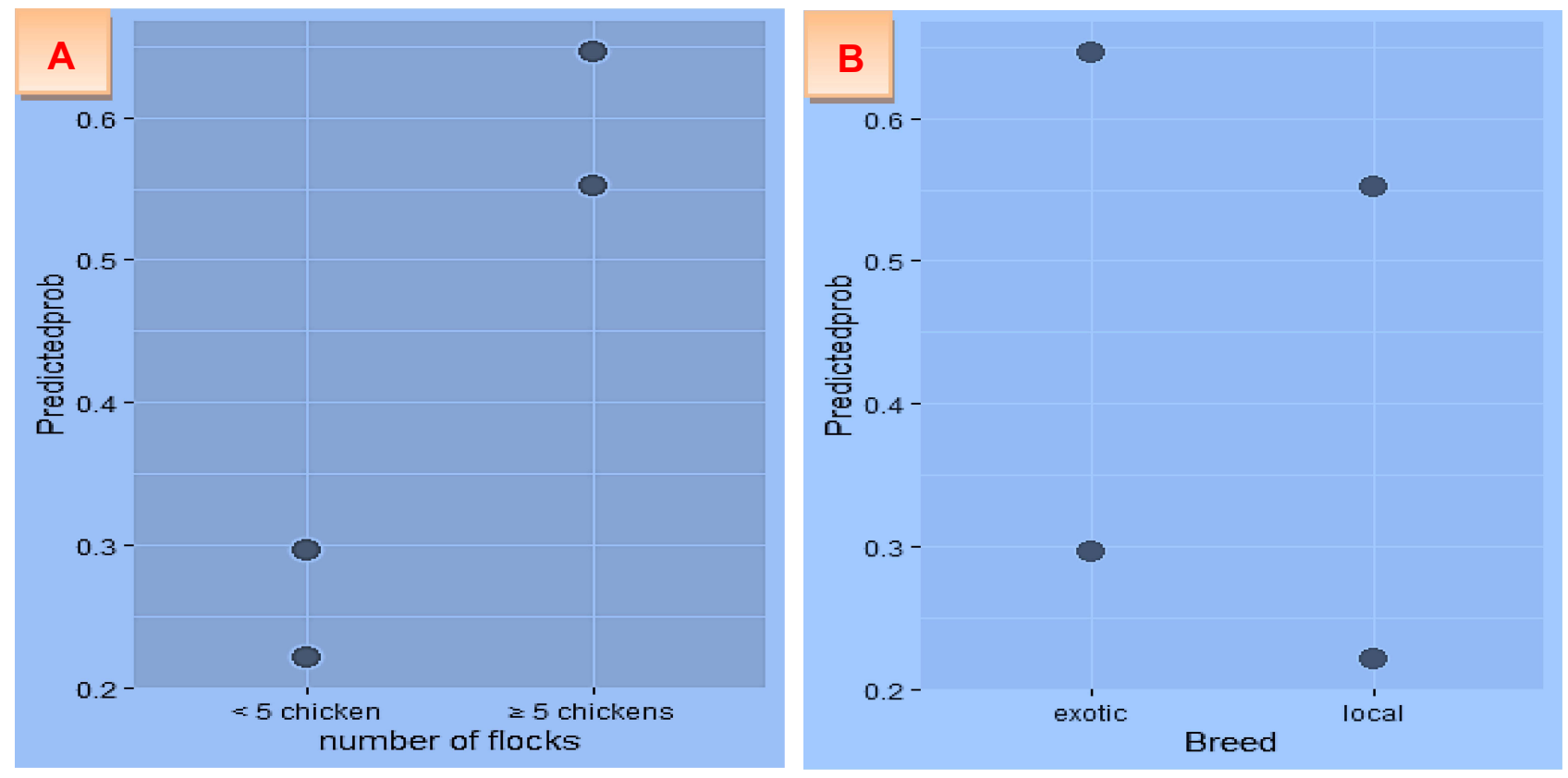

Figure 2 Ggplot of predicted probability of the occurrence IBD in association with the flock size (A) and breed (B).

houses. The result of the production features disclosed by the respondents is given in Table 2.

None of the chicken owners had their chicken vaccinated. Half of the respondents (50\%) had information about IBD. Six $(10 \%)$ of the interviewed chicken owners revealed that their chickens had contact with the wild birds and $66 \%$ of them disclosed that diseases and predators are the standing problems affecting chicken production in the area. All of the owners $(100 \%)$ claim that housing and feed shortage are among major obstacles to the improvement of chicken production.

Table 2 Types of Poultry Production, Socioeconomic Importance, and Management System Practiced

\begin{tabular}{|l|l|r|}
\hline Variables & Frequency & Percent (\%) \\
\hline $\begin{array}{l}\text { Types of poultry productions } \\
\text { Layer }\end{array}$ & 7 & \\
Broiler & 9 & 11.66 \\
Both & 44 & 15.00 \\
Socio-economic importance & & 73.33 \\
Home consumption & 5 & \\
Primary source of income & 6 & 8.33 \\
Secondary source of income & 49 & 10.00 \\
Management practice & & 81.66 \\
Supportive feed provided & 30 & \\
Dwell at home & 47 & 50.00 \\
Lives in a separate house & 13 & 78.33 \\
Cleaning poultry house & 60 & 21.66 \\
Burn and bury wastage & 26 & 100.00 \\
Throw away in open space & 34 & 43.33 \\
\hline
\end{tabular}

Among the chicken owners interviewed $58.30 \%$ had no practice of treating or seeking veterinary services for sick chickens; $84.00 \%$ of those who did not treat or seek veterinary services revealed mortality in their chickens. Only $21.66 \%$ of the respondents reported the practice of isolation of sick chickens; $63.30 \%$ prove that there was an IBD outbreak previously in the study areas among them $77 \%$ of respondents had a lack of practice of isolating sick chickens (Table 3). There was a statistically significant association $(\mathrm{P}<0.05)$ between treatment history and isolation of sick chickens with mortality in the flock. The previous outbreaks had also a statistically significant association $(\mathrm{P}<0.05)$ with the isolation of sick chickens in the flock.

\section{Discussion}

In Ethiopia, chicken production remains an important economic activity, serving the community as a cheap source of quality protein, cash, and social capital. In its attempt to achieve food security, the Ethiopian government has made chicken production a priority sector. The goal is to improve the number of family poultry production along with small and large-scale commercial poultry farms. ${ }^{15}$ However, the goal will remain ambition in the absence of control of infectious diseases including IBD. Understanding the epidemiology of IBD in each production system is important for its control. Thus, this study provided information on the occurrence of IBD in backyard chicken in the Wolaita zone where 
Table 3 Previous Outbreak and Death History in Association with Treatment and Isolation of Sick Chickens

\begin{tabular}{|l|c|c|c|c|c|c|}
\hline \multirow{2}{*}{ Variables } & \multicolumn{3}{|c|}{ Death in the Flock } & \multicolumn{3}{c|}{ Previous IBD Outbreaks } \\
\cline { 2 - 6 } & Yes (\%) & No (\%) & P-value & Yes (\%) & No (\%) & P-value \\
\hline Treated & $6(17.1 \%)$ & $29(82.9 \%)$ & $0.001 *$ & $19(54.3 \%)$ & $16(45.7 \%)$ & 0.147 \\
Not treated & $21(84.0 \%)$ & $4(16.0 \%)$ & & $19(76.0 \%)$ & $6(24.0 \%)$ & $10(77.0 \%)$ \\
Sick chickens isolated & $1(7.7 \%)$ & $12(92.3 \%)$ & $0.006 *$ & $3(23.0 \%)$ & $0.002 *$ \\
Sick chickens not isolated & $26(55.3 \%)$ & $21(44.7 \%)$ & & $35(74.5 \%)$ & $12(25.5 \%)$ & \\
\hline
\end{tabular}

Note: *Represent statistically significant.

chicken production plays a key role in the livelihoods of the community. We demonstrated the widespread occurrence of anti-IBDV antibodies, which in the absence of vaccination, showed natural exposure to the virus. The anti-IBDV antibody was detected in chickens tested from all four districts in the zone irrespective of their agroecology. The IBDV is resistant to various environmental conditions and can survive longer in the environment, ${ }^{3}$ revealing that agroecology is a limiting factor for the incidence of the disease. This is also supported by the results of the questionnaire, which showed that the interviewed farmers rated IBD to be among the top constraints to chicken production. The widespread occurrence of IBD observed in this study is attributed to the scavenging characteristics of the chickens in which there is free movement and contact among nearby flocks and poor knowledge of the farmers about the disease and biosecurity measures.

The seroprevalence of IBD observed in this study is high. This will have a significant effect on the establishment and expansion of commercial poultry farms as the backyard chicken could serve as a reservoir of IBDV for the chicken. This is in agreement with the previous seroprevalence report in backyard chicken by Zegeye et al ${ }^{16}$ from Mekelle with a seroprevalence of $45 \%$ and Lemma et $\mathrm{a}^{17}$ from Jigjiga and Harar districts with a seroprevalence of 52\%. A higher prevalence than our observation has also been reported in various parts of Ethiopia. ${ }^{11,18}$ The difference observed in the seroprevalence between our findings and that of the previous studies is due to variation in the nature of samples and serological tests used. For example, Jenbreie et $\mathrm{al}^{11}$ test the serum samples collected during and immediately after outbreaks of IBD. The sensitivity of a serological test used is also an important factor, ${ }^{4}$ where a less sensitive ELISA kit was utilized than the current study. All these observations suggest that IBD became endemic in backyard chicken in many parts of Ethiopia warranting the need for attention by the veterinary and livestock authorities.
This study revealed that indigenous chicken ecotypes (local breeds) had lower odds of being infected with IBDV compared to the exotic chicken breeds reared in the area. In other words, the exotic chicken breed had higher odds of being infected than the indigenous chickens. This result is consistent with earlier findings of Jenbreie et $\mathrm{al}^{11}$ and Zeryehun and Fekadu ${ }^{12}$ who reported higher seroprevalence of IBD in exotic chickens than local breeds. This could be due to the innate resistance of the indigenous chickens due to long-term exposure and co-evolution with the virus as there are no biosecurity measures under backyard production systems. In contrast, the exotic chicken was imported from abroad where there are strict biosecurity measures that minimize the exposure of the chickens to the virus. The selection of the chickens for productivity traits also increases their susceptibility to diseases.

The number of flocks owned per household is also an important risk factor of seroprevalence of IBD in the study area. Statistically significantly higher seroprevalence was observed in chickens tested from households keeping $\geq 5$ chickens $(\mathrm{OR}=4.07 ; \mathrm{P}<0.001)$. Previous studies showed that flock size has significant effects on the occurrence of disease. ${ }^{19,20}$ The higher seroprevalence in chickens from larger flock sizes is due to increased contact rate and density, which favors the spread of the virus among the chickens. This is further evident for the questionnaire survey, which uncovered a statistically significant association between treatment history, isolation of sick chickens, the occurrence of death in the flock, and seroprevalence of IBD. Moreover, the history of previous outbreaks had a statistically significant association with the isolation of sick chickens in the flock and the occurrence of IBD. This suggests the lack of knowledge about the epidemiology of the disease by the farmers, which ultimately results in the continual transmission of the virus in the area. Thus, the higher the flock size, the higher chance of being exposed to the virus. 


\section{Conclusions}

The present study revealed a higher seroprevalence of IBD in traditionally managed chickens in Wolaita Sodo with an overall prevalence of $48.96 \%$. Flock size and breed of chickens were identified as important risk factors for the occurrence of IBD in the area. The results of the questionnaire showed that chicken producers did not have enough knowledge about the nature and epidemiology of IBD. Proper management practices together with appropriate vaccination programs are necessary to reduce IBD incidence in the study areas. Veterinary and livestock authorities should take the occurrence of IBD into account in their endeavors to improve chicken production in the area.

\section{Data Sharing Statement}

The data collected and used to support this article can be offered by the first or corresponding author upon request.

\section{Ethical Approval and Consent to Participate}

Ethical approval for this study was granted from the animal research ethical review committee of the College of Veterinary Medicine and Agriculture of Addis Ababa University (Reference number: VM/ERC/22/06/13/2021). Animals were handled according to OIE guiding principles for animal welfare. The chicken owners, which were included in this study, were informed about the purpose and benefits of the study, and verbal consent from each participant was obtained because they are unable to write and read. Consents were taken in the presence of a third independent party and approved by the College of Veterinary Medicine and Agriculture of Addis Ababa University Ethics Committee.

\section{Acknowledgments}

The authors would like to acknowledge the National Animal Health Diagnostic and Investigation Center (NADIC) for the provision of laboratory facilities and Addis Ababa University Thematic Research Project Number: VPRTT/LT-497/2019 for the financial support provided. Chicken owners and households were highly acknowledged for their unreserved support during sample and data collection.

\section{Funding}

This study was supported by the Addis Ababa University Thematic Project number: VPRTT/PY- VPRTT/LT-497/
2019, Ethiopia. The funder had no role in the conception, design of the study, data collection, analysis, and interpretation of the data reported in this manuscript.

\section{Disclosure}

The authors declare that they have no competing interests in this work.

\section{References}

1. Birol E, Asare-marfo D, Ayele G, et al. Investigating the role of poultry in livelihoods and the impact of avian flu on livelihoods outcomes in Africa: evidence from Ethiopia, Ghana, Kenya, and Nigeria. IFPRI discussion papers 1011. International Food Policy Research Institute (IFPRI); 2010.

2. Choudhary UK, Tiwary BK, Prasad A, Ganguly S. Study on incidence of infectious bursal disease in and around Ranchi. Indian J Anim Res. 2012;46(2):156-159.

3. Camilotti E, Moraes L, Furian T, Borges K, Moraes H, Salle C. Infectious bursal disease: pathogenicity and immunogenicity of vaccines. Braz J Poult Sci. 2016;18(2):303-308. doi:10.1590/18069061-2015-0148

4. OIE. Infectious bursal disease (Gumboro disease). In: OIE Terrestrial Manual. Vol. 148; 2018:148-162.

5. Brandt M, Yao K, Liu M, Heckert RA, Vakharia VN. Molecular determinants of virulence, cell tropism, and pathogenic phenotype of infectious bursal disease virus. J Virol. 2001;75(24):11974-11982. doi:10.1128/JVI.75.24.11974-11982.2001

6. Müller H, Islam R, Raue R. Research on infectious bursal disease the past, the present and the future. Vet Microbiol. 2003;97:153-165. doi:10.1016/j.vetmic.2003.08.005

7. Sharma JM, Kim I, Rautenschlein S, Yeh H. Infectious bursal disease virus of chickens: pathogenesis and immunosuppression. Dev Comp Immunol. 2000;24:223-235. doi:10.1016/S0145-305X(99)00074-9

8. Orakpoghenor O, Oladele SB, Abdu PA. Infectious bursal disease: transmission, pathogenesis, pathology and control - an overview. Worlds Poult Sci J. 2020;76(2):292-303. doi:10.1080/ 00439339.2020.1716652

9. Zeleke A, Gelaye E, Sori T, Ayelet G, Sirak A, Zekarias B Investigation on infectious bursal disease outbreak in Debre Zeit, Ethiopia. Int J Poult Sci. 2005;4(7):504-506. doi:10.3923/ ijps.2005.504.506

10. Jenberie S, Lynch SE, Kebede F, et al. Genetic characterisation of infectious bursal disease virus isolates in Ethiopia. Acta Trop. 2014;130:39-43. doi:10.1016/j.actatropica.2013.09.025

11. Jenbreie S, Ayelet G, Gelaye E, Kebede F, Lynch SE, Negussie H. Infectious bursal disease: seroprevalence and associated risk factors in major poultry rearing areas of Ethiopia. Trop Anim Health Prod. 2012;45(1):75-79. doi:10.1007/s11250-012-0176-3

12. Zeryehun T, Fekadu G. Seroprevalence of infectious bursal disease in chickens managed under backyard production system in Central Oromia, Ethiopia. Afr J Microbiol Res. 2012;6(38):6736-6741. doi:10.5897/AJMR12.1344

13. Woldemariam S, Wossene A. Infectious bursal disease (Gumboro Disease): case report at Andasa poultry farm, Amhara region. Ethiop Vet J. 2007;11(1):151-155.

14. Thrusfield M. Veterinary Epidemiology. 3rd ed. London: Black Well Science Ltd; 2005.

15. Shapiro B, Gebru G, Desta S, et al. Ethiopia livestock master plan roadmaps for growth and transformation. ILRI Project Report. Nairobi, Kenya: International Livestock Research Institute (ILRI); 2015 . 
16. Zegeye S, Tsegaye Y, Abreha H, Awol N. Sero-prevalence of infectious bursal disease in backyard chickens around Mekelle, Northern Ethiopia. Afr J Biotechnol. 2015;14(5):434-437. doi:10.5897/ AJB2014.14349

17. Lemma F, Zeryehun T, Kebede A. Seroprevalence of infectious bursal disease in non-vaccinated village chicken in Jigjiga and Harar districts, Eastern Ethiopia. J Vet Sci Technol. 2019;10(1):6-10.

18. Chaka H, Goutard F, Bisschop SPR, Thompson PN. Seroprevalence of Newcastle disease and other infectious diseases in backyard chickens at markets in Eastern Shewa zone, Ethiopia. Poult Sci. 2012;91:862-869. doi:10.3382/ps.2011-01906
19. Rashid H, Xue C, Islam T. Risk factors associated with infectious bursal disease in commercial chickens in Bangladesh. Prev Vet Med. 2013;111(1-2):181-185. doi:10.1016/j.prevetmed.2013.03.013

20. Farooq M, Durrani FR, Faisal S. Incidence of infectious bursal disease among birds submitted to a diagnostic laboratory in NWFP, Pakistan. Pak Vet J. 2000;20(2):77-80.

\section{Publish your work in this journal}

Veterinary Medicine: Research and Reports is an international, peerreviewed, open access journal publishing original research, case reports, editorials, reviews and commentaries on all areas of veterinary medicine. The manuscript management system is completely online and includes a very quick and fair peer-review system. Visit http://www.dovepress.com/testimonials.php to read real quotes from published authors. 